\title{
Online Intelligent Identification of Modal Parameters for Large Cable-Stayed Bridges
}

\author{
Peng Wen, ${ }^{1}$ Inamullah Khan ${ }^{D},{ }^{2}$ He Jie, ${ }^{1}$ Chen Qiaofeng, ${ }^{1}$ and Yang Shiyu ${ }^{1}$ \\ ${ }^{1}$ Bridge Engineering Department, Southwest Jiaotong University, Chengdu, China \\ ${ }^{2}$ School of Civil Engineering, National University of Sciences and Technology, Islamabad, Pakistan \\ Correspondence should be addressed to Inamullah Khan; inam_bunny@yahoo.com
}

Received 17 August 2019; Accepted 19 December 2019; Published 18 February 2020

Academic Editor: Sakdirat Kaewunruen

Copyright (c) 2020 Peng Wen et al. This is an open access article distributed under the Creative Commons Attribution License, which permits unrestricted use, distribution, and reproduction in any medium, provided the original work is properly cited.

Realizing online intelligent identification of bridge modal parameters requires not only the adaptive decomposition of structural response signals but also the enforcement of the automatic identification of modal parameters. Therefore, in this paper, the signal decomposition algorithm-ensemble empirical mode decomposition algorithm (EEMD) is improved to fulfill the above task. First, the adaptive matching algorithm is introduced to deal with the endpoint effect; second, the method of classification is used to avoid modal aliasing. Finally, an index for filtering the effective intrinsic mode function (IMF) components is constructed to realize automatic screening and signal reconstruction of the effective IMF components. At the same time, the first derivative of the singular entropy increment is used to automatically determine the order of the system, and then the spectral clustering algorithm is combined with the stochastic subspace algorithm to ultimately reach the goal of automatic identification of modal parameters.

\section{Introduction}

A bridge structure health monitoring system is important to guarantee the operational safety of existing bridge structures, and the modal parameter identification [1] of bridge structures is an important aspect of a bridge health monitoring system. The accurate identification of these parameters can reflect the inherent dynamic characteristics of the bridge structure and can aid in the long-term health monitoring of the bridge. However, the existing modal parameter identification algorithms cannot automatically identify parameters and still require human participation to determine the order of the system [2] and evaluate stability diagrams [3]. Additionally, the dynamic signals of bridge structures under environmental excitation generally contain noise, and the various sources of the noise are complex. The noise in a vibration signal can be reduced in the data acquisition stage by taking measures such as averaging, filtering, and shielding [4]. However, it is impossible to eliminate the noise altogether. In view of this limitation, to obtain more accurate bridge modal parameters during bridge system assessment and improve the damage diagnosis and health monitoring of structures, it is necessary to preprocess the response signals of bridge structures under environmental excitation conditions, that is, the signal must be decomposed and reconstructed. Based on this approach, in this paper, the existing ensemble empirical mode decomposition (EEMD) algorithm [5] is improved, and then a statistics-based spectral clustering algorithm [6] is combined with a stochastic subspace identification method [7] for the online automatic identification of modal parameters.

\section{Improvement of the EEMD}

The EEMD algorithm [5] is an important improvement to the original empirical mode decomposition (EMD) algorithm. The specific method involves repeatedly adding random white noise of equal amplitude to the original signal to homogenize the specific distribution of the extreme value points in the signal. Therefore, the influence of the intermittent high-frequency component is avoided to some extent. In addition, to counteract the white noise in the intrinsic mode function (IMF), it is necessary to obtain the ensemble mean of the results and retain the IMF component, 
which has physical meaning. The specific implementation steps are shown in the literature [5].

Based on the core principle of the algorithm, the method still has the following limitations: the processing effect of endpoints is poor, the obtained intrinsic mode function still includes mode aliasing, and selecting effective IMF components for signal reconstruction is difficult. Considering the above three problems, this paper proposes a corresponding improved algorithm to cope with these problems effectively.

2.1. Addition of Positive and Negative White Noise. In the process of EEMD, the white noise signal should be added to the original signal, which is then decomposed. In this approach, signal components with different time scales can be automatically decomposed into the pass band, which is determined by the white noise signal, to overcome the mode mixing phenomenon [8]. However, due to the addition of the white noise signal to the original signal, the white noise signal will disrupt the original signal, which will reduce the signal-to-noise ratio (SNR) of the decomposition results. Because the negative effect of white noise cannot be completely eliminated, the reconstruction error of this algorithm is large, and the completeness of the decomposition is poor.

Based on these factors, in this paper, positive and negative white noise sequences are added to the original signal to solve the above problems.

The specific steps in this method are as follows:

(1) Addition of random white noise to the original signal $s(t)$, including $K$ sets of positive white noise $n^{i}(t)$ and $K$ sets of negative white noise $-n^{i}(t)$. The calculation formula is as follows:

$$
x(t)=s(t)+(-1)^{q} a_{0} n^{i}(t),
$$

where $q=1$ or 2 (equal to 1 if negative white noise is added and equal to 2 if positive white noise is added), $i=1,2, \ldots, K, K$ represents the total number of times that white noise is added, $a_{0}$ represents the standard deviation of the amplitude of the added white noise, $n^{i}(t)$ represents the white noise that is added for the $i^{\text {th }}$ time, $s(t)$ represents the original signal, and $x(t)$ represents the signal after the white noise is added.

(2) The mixed signal with positive and negative paired white noise is an input into the EEMD algorithm and then decomposed.

In practical applications, the amplitude of the added white noise cannot be too large or too small; if the amplitude is too large, the noise will cover up the effective signal, and the residual noise will be difficult to eliminate. However, if the amplitude is too small, the distribution of extreme points in the original signal may not be sufficiently disrupted, and eliminating the phenomenon of mode aliasing cannot be achieved. According to a previous study [8], the amplitude of the added white noise is usually 0.1-0.2 times the standard deviation of the original signal.

\subsection{Adaptive Extreme Point-Matching Continuation} Algorithm. To solve the endpoint effect problem in the EEMD algorithm, many scholars have proposed improved methods, which can be roughly divided into the following two categories:

(1) The extreme point continuation method [9]: the basic principle of this method involves extending the original signal data to a certain number of extreme points. One of the main advantages of this algorithm is that it is simple and easy to implement. However, the limitation is that this approach only considers the partial information of the signal at an endpoint and does not consider the signal as a whole.

(2) Predictive continuation method [10]: in this method, the original signal is predicted and extended using a neural network [11], ARMA model [12], or support vector machine method [13]. The effectiveness of these algorithms mainly depends on the parameter values because the approach is not adaptive and thus requires many computations and has a long operation time.

Combining the advantages and disadvantages of the above two methods, a new continuation method is proposed: an adaptive extreme point-matching continuation algorithm. The main feature of this algorithm is that it can determine the best-matched extreme points from inside the original signal to match with the extreme points at both ends of the signal. This approach extends the signal considering the original trend in a rapid manner and is highly adaptable.

The basic principles of the algorithm are as follows.

Set the signal equal to $x(t)$. Additionally, $m_{i}, n_{i}(i=1,2,3, \ldots)$ are the maximum and minimum points, respectively, and the corresponding times are $t_{m i}$ and $t_{n i}$. Taking the extreme left point continuation of $x(t)$ as an example, $x_{t x 1}$ is the starting point at the left, and $x_{t x 1}-m_{1}-$ $n_{1}$ form an extreme point characteristic wave. Then, the extreme point waveform that best matches the characteristic wave is determined from the signal. In this case, $x_{t x i}-m_{i}-$ $n_{i}$ is the matching extreme point wave, and the matching extreme point is extended to the left endpoint of the original signal (the right end is extended to the right endpoint).

Then, the next specific steps in this method are as follows.

Obtain all $x_{t x i}$ values except the left endpoint $x_{t x 1}$. The corresponding time value can then be obtained from the similarity relation.

$$
t_{x i}=\frac{t_{m 1} t_{m i}-t_{n 1} t_{n i}}{t_{m 1}-t_{n 1}},
$$

where $t_{m 1}$ is assumed to be less than $t_{n 1}$. If the obtained value of $t_{x i}$ is not a sampling point, the specific value of $x_{t x i}$ can be obtained by linear interpolation.

Calculate the error $e$ between the obtained extreme points and the extreme points of the feature. 


$$
e(i)=\frac{\left|m_{i}-m_{1}\right|}{\left|m_{1}\right|}+\frac{\left|n_{i}-n_{1}\right|}{\left|n_{1}\right|}+\frac{\left|x_{t x i}-x_{t x 1}\right|}{\left|x_{t x 1}\right|} .
$$

In the formula, the reason for dividing by the first maximum, the first minimum, and the signal endpoint value is to standardize the error term.

Determine the minimum matching error $e(i)_{\min }$. The extreme point is the matched extreme point at a given time, and the data associated with this matched extreme point are shifted to the left end of the original signal as the continuation extremum.

According to the steps followed below, the right endpoint of the original signal is matched and extended, and the last extended signal is set to $\tilde{x}(t)$.

Then, decomposition of the extended signal $\tilde{x}(t)$ is performed and the IMF components are obtained according to the timing of the original signal. In this approach, the decomposition results by considering the improved endpoint can be obtained.

The aforementioned continuation algorithm not only considers the trend and regularity of the signal but also only requires the extension of extreme points. This process is easy to implement and is more self-adaptable as compared to other methods.

Currently, the direct observation method is generally used to assess the processing effect of the signal endpoints. Based on this method, an evaluation index $\theta$ is introduced to evaluate the treatment of endpoints. The specific calculation process is as follows.

First, the effective RMS of the original signal and each IMF component are calculated and used to estimate the energy of each signal sequence. The specific calculation formula of the RMS is as follows:

$$
\mathrm{RMS}=\sqrt{\frac{\sum_{i=1}^{n} S^{2}(i)}{n}},
$$

where $S(i)$ is the signal sequence, that is, the original signal $x(t)$ of each IMF component, and $n$ is the number of samples in the signal.

According to formula (4), the sum of the effective values of each IMF component and the effective value of the original signal is calculated and compared, and then the evaluation index $\theta$ is obtained.

$$
\theta=\frac{\left|\sum_{f=1}^{k} \mathrm{RMS}_{f}-\mathrm{RMS}_{x}\right|}{\mathrm{RMS}_{x}},
$$

where $\mathrm{RMS}_{x}$ is the effective value of the original signal, $\mathrm{RMS}_{f}$ is the valid value of the $f^{\text {th }}$ IMF component, and $k$ is the total number of IMF components, including the residuals in the decomposed signal.

From the definition, there is no endpoint effect if $\theta=0$, and the larger the value is, the greater will be the endpoint effect.

2.3. Mode Aliasing Process. When the signals are decomposed by the EEMD algorithm, the phenomenon of modal aliasing may influence the obtained IMFs, that is, similar characteristic time scales are distributed among different IMF components. As a result, the waveforms of two adjacent IMF components are mixed together, thus making it difficult to identify each signal. To avoid this phenomenon, a clustering analysis algorithm [14] is introduced. However, before analyzing the clustering algorithm, it is necessary to understand the basic process of the EEMD algorithm. The flowchart is shown in Figure 1.

According to the flowchart, the final IMF is obtained from the ensemble average of $N$ numbers of $\mathrm{IMF}_{s}$ after EEMD decomposition. The calculation formula is as follows:

$$
\mathrm{IMF}_{j}=\frac{1}{N} \sum_{i=1}^{N} \mathrm{IMF}_{i j}
$$

where $\mathrm{IMF}_{j}$ is the $j^{\text {th }}$ IMF obtained from the original signal, which is decomposed by the EEMD algorithm.

The principle of the EEMD algorithm suggests that the EEMD algorithm only directly takes the average value of $\operatorname{IMF}_{i j}(i=1,2, \ldots, N)$ when calculating $\operatorname{IMF}_{j}$ but does not consider whether the $N \operatorname{IMF}_{i j}(i=1,2, \ldots, N)$ values in the same line belong to the same class. At the same time, it is possible for mode aliasing to exist between $n-1 \mathrm{IMF}_{i j}(j=$ $1,2, \ldots, n-1)$ values in the same column. Based on this approach, a clustering analysis method [15] involving multivariate data analysis is introduced to solve the above problems, and the specific process is as follows:

(1) In the modal decomposition of the signal, each decomposition yields a series of components. To ensure that there is no aliasing among these obtained components, clustering analysis can be performed. If there is mode aliasing among the obtained components, the results for the relevant signals are eliminated, and then white noise is added again. This process is repeated until there is no mode aliasing in the decomposition results.

(2) A clustering analysis was performed on $N \mathrm{IMF}_{i j}$ values in the same row to select the class of IMF with the largest number of clusters. Then, the average value is established as the final $\mathrm{IMF}_{j}$.

Introducing the clustering analysis algorithm into the EEMD algorithm not only guarantees that there is no mode aliasing among IMF components in each decomposition but also guarantees that there is no mode aliasing among the final IMFs.

2.4. Filtering of Effective IMF Components. When the signal is decomposed by the EEMD algorithm, multiple intrinsic mode functions $\left(\mathrm{IMF}_{s}\right.$ ) can be obtained, but the effective IMF components cannot be automatically filtered. In practice, it is often necessary to manually participate in the screening of effective IMF components according to the Hilbert-Huang spectrum of each IMF component, thereby reducing the work efficiency and leading to subjectivity in the selected results due to individual differences.

Based on this limitation, an algorithm for the automatic screening of effective IMF components is proposed. This 


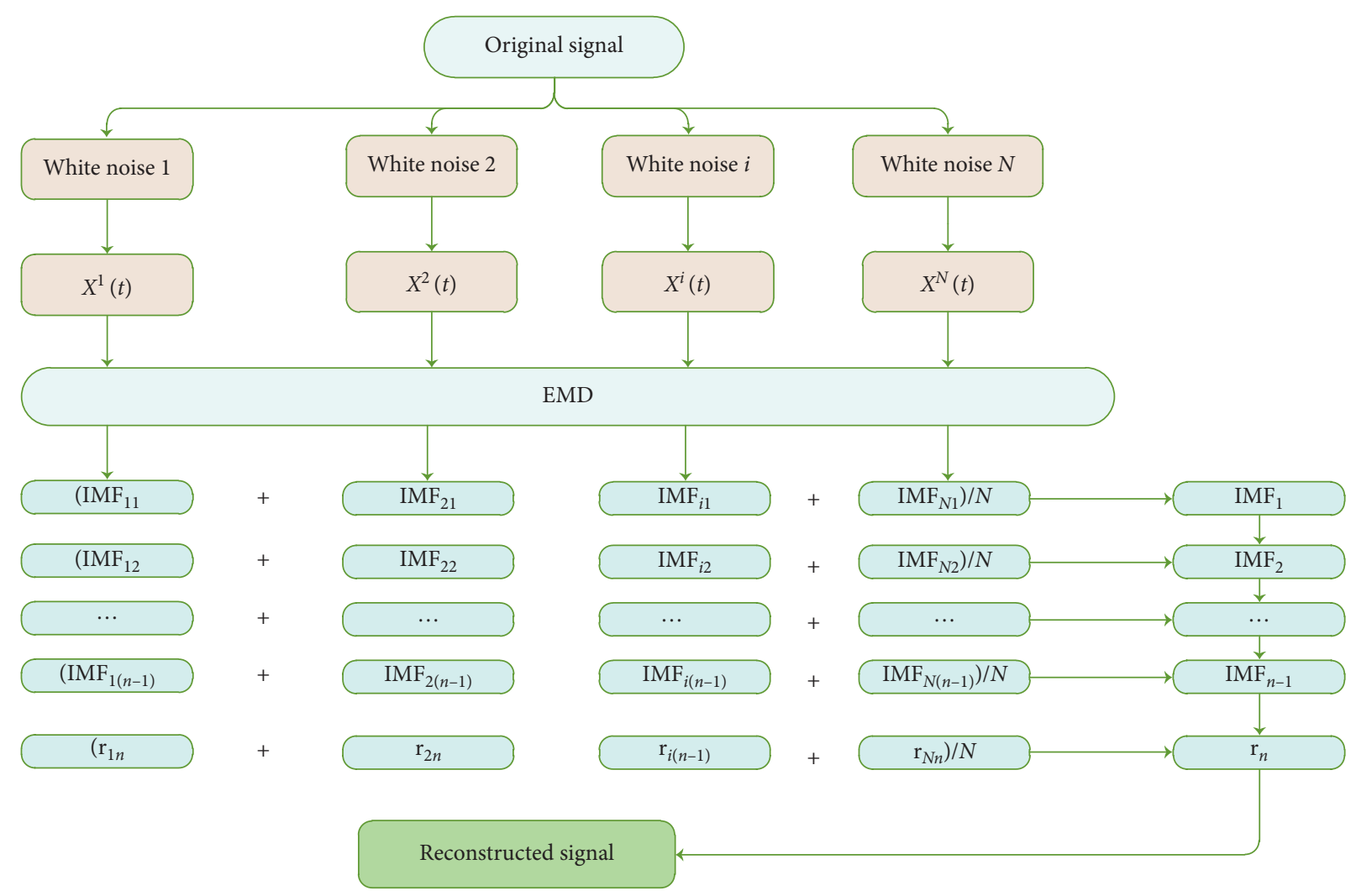

FIgure 1: Flowchart of the EEMD algorithm.

algorithm not only considers the information entropy [16] of each IMF component but also merges the energy density and average period [17]. It is necessary to introduce the information entropy, the energy density, and the average period of the IMF components before the filtering algorithm is presented.

2.4.1. The Information Entropy of IMF Components. A series of components with different bandwidths can be obtained by the mode decomposition of the response signal. Each component contains different frequencies of the signal from high to low, and these frequencies and bandwidths will change as the signal changes. In addition, studies of information entropy have indicated that the more orderly the signal components contained in the IMF are and the better the aggregation of the time-frequency distribution is, the smaller the resulting value of information entropy is. In contrast, the more disorderly the signal is and the worse the aggregation of the time-frequency distribution is, the greater the value of the resulting information entropy is.

The following steps detail how to calculate the information entropy of each IMF component:

(a) It is assumed that $f(t)$ is a set of components of the obtained IMF after the decomposition of signal $x(t)$. The maximum value of $f(t)$ is $f_{\max }$, and the minimum value is $f_{\text {min }}$.

(b) $A_{i}$ is set equal to $N$ within the interval $\left[f_{\min }, f_{\max }\right]$, and the interval $\left[f_{\min }, A_{1}\right],\left(A_{1}, A_{2}\right], \ldots,\left[A_{N-1}, f_{\max }\right)$ is the discrete range $B \in\left\{B_{1}, B_{2}, \ldots, B_{N}\right\}$ of the characteristic quantity. When the value of a sample with a qualifying attribute falls into the interval $\left(A_{i}, A_{i+1}\right.$ ], this sample has the corresponding discrete attribute value $B_{i}$.

(c) When the total number of sampling points for the discrete signals is $n$, the number of sample points of $f(t)$ that fall in the $i^{\text {th }}$ interval is $m_{i}$. Thus, the probability $P\left(B_{i}\right)=m_{i} / n$ of $f(t)$ falling within the $i^{\text {th }}$ interval can be calculated according to the corresponding statistics. Therefore, according to the third definition [16] of information entropy, the information entropy of the IMF can be calculated as follows:

$$
H=-\sum_{i=1}^{N} p\left(x_{i}\right) \log P\left(x_{i}\right) .
$$

2.4.2. Energy Density and Average Period. According to reference [17], for a signal with a white noise sequence, the product of the energy density and the average period of each component is a constant when EMD is used to decompose the signal. Therefore, an algorithm for filtering components is proposed. The specific process is as follows.

First, the mode decomposition of the response signal is performed, and the energy density $(E)$ and average period $(\bar{T})$ corresponding to each IMF component are calculated. Next, the product of these two components is calculated and 
is defined as a mathematical expression of the energy coefficient $(E T)$ as follows:

$$
E_{j}=\frac{1}{N} \sum_{i=1}^{N}\left(f_{j}(i)\right)^{2} \bar{T}_{j}=\frac{2 N}{O_{j}},
$$

where $N$ is the length of the original signal, $f_{j}$ is the amplitude of the $j^{\text {th }}$ IMF component, and $O_{j}$ is the number of existing extreme points for the $j^{\text {th }}$ IMF component.

The energy coefficient (ET) of each IMF component is used to calculate the final effective coefficient RP of each IMF component. The calculation formula is as follows:

$$
\mathrm{RP}_{j}=\left|\frac{E T_{j}-(1 / j-1) \sum_{i=1}^{j-1} E T_{i}}{(1 / j-1) \sum_{i=1}^{j-1} E T_{i}}\right| .
$$

When the effective coefficient $\mathrm{RP}_{j}$ of the $j^{\text {th }}$ IMF component is greater than or equal to 1 , the energy coefficient corresponding to the $j^{\text {th }}$ IMF component exponentially increases compared with the average value of the previous $j-1^{\text {th }}$ IMF component. In this case, the product of the energy density of the previous $j-1^{\text {th }}$ IMF component and the average period can be considered a constant, that is, the previous $j-1^{\text {th }}$ IMF component can be regarded as noise and deleted. Therefore, what is left is the effective component.

2.4.3. Comprehensive Evaluation Algorithm. If the effective IMF is only selected according to the information entropy of each IMF component, there may be errors in the selection results. Therefore, a comprehensive evaluation algorithm [18] is proposed by combining the information entropy $(H)$ and the effectiveness coefficient (RP). This algorithm is a type of comprehensive bid evaluation method based on fuzzy mathematics and mainly transforms a qualitative evaluation into a quantitative evaluation according to the membership theory of fuzzy mathematics. A new index (effectiveness degree) can be constructed using the information entropy and the effectiveness coefficient to quantify the effectiveness degree $Y$ between each IMF component and the original signal.

The specific formula is as follows:

$$
Y_{i}=w_{H}\left(1-\frac{H_{i}-H_{\text {min }}}{H_{\text {max }}-H_{\text {min }}}\right)+w_{\mathrm{RP}}\left(\frac{\mathrm{RP}_{i}-\mathrm{RP}_{\text {min }}}{\mathrm{RP}_{\text {max }}-\mathrm{RP}_{\text {min }}}\right),
$$

where $w_{H}$ is the weight of the information entropy $H$ and $w_{\mathrm{RP}}$ is the weight of the effectiveness coefficient RP.

In the literature [19], a processing method for determining the weight value is given. Considering the primary and secondary relations between information entropy and the effectiveness coefficient, it is assumed that both weight coefficients in formula (10) are 0.5 .

The above analysis shows that the closer $Y_{i}$ is to 1 , the higher the degree of association is between the $i^{\text {th }}$ component and the original signal. In combination with reference [20], this paper assumes that when $Y \geq 0.8$, the IMF component is considered effective. Finally, summing all effective
IMF components, the final reconstruction signal can be obtained.

The flowchart of the improved EEMD algorithm is given in Figure 2.

\section{Improvement of the Stochastic Subspace Identification Algorithm}

Currently, the stochastic subspace identification (SSI) [21] algorithm is a commonly used structural modal parameter identification algorithm. This method is mainly used in linear systems and is a type of time domain identification method, as compared with other modal parameter identification methods. This algorithm does not require a parameterized system model but adopts basic matrix analysis methods such as QR decomposition and SVD decomposition. However, this algorithm still has the following two limitations:

(1) The real order of the system is difficult to determine, and there is no unified method or theory to determine the real order of the system.

(2) Currently, the selection of extreme points in the stability diagram often requires manual participation in the selection. However, in practice, because different points are manually selected, the final modal parameter identification results can differ. Based on the above two problems, a new system order determination algorithm and a real modal filtering algorithm are proposed.

3.1. Automatic Determination of the System Order. The most commonly used order determination method is the stability diagram, but it still has two limitations:

(1) Modal distortion is mainly caused by external factors that do not belong to the system itself or the mode of the system itself cannot be identified due to this distortion.

(2) The large number of calculations is the main reason why stability diagram theory is based on structural parameters such as the frequency, mode shape, and damping as the basis for determining the stability point. Therefore, it is necessary to calculate a large number of modal parameters for a structure of given order. Based on this requirement, singular entropy theory is introduced to solve the problem of determining order difficulty. The algorithm does not need to use the modal parameters of the structure to determine the stability point, which can greatly reduce the calculation workload.

A detailed description of how to use the singular entropy to determine the system order is given as follows.

By analyzing the decomposition principle of the singular value of the signal [22], a real matrix $Q$ with dimensions of $m \times n$ can be decomposed into a matrix $R$ with dimensions of $m \times l$, a diagonal matrix $\Lambda$ with dimensions of $l \times l$, and a 


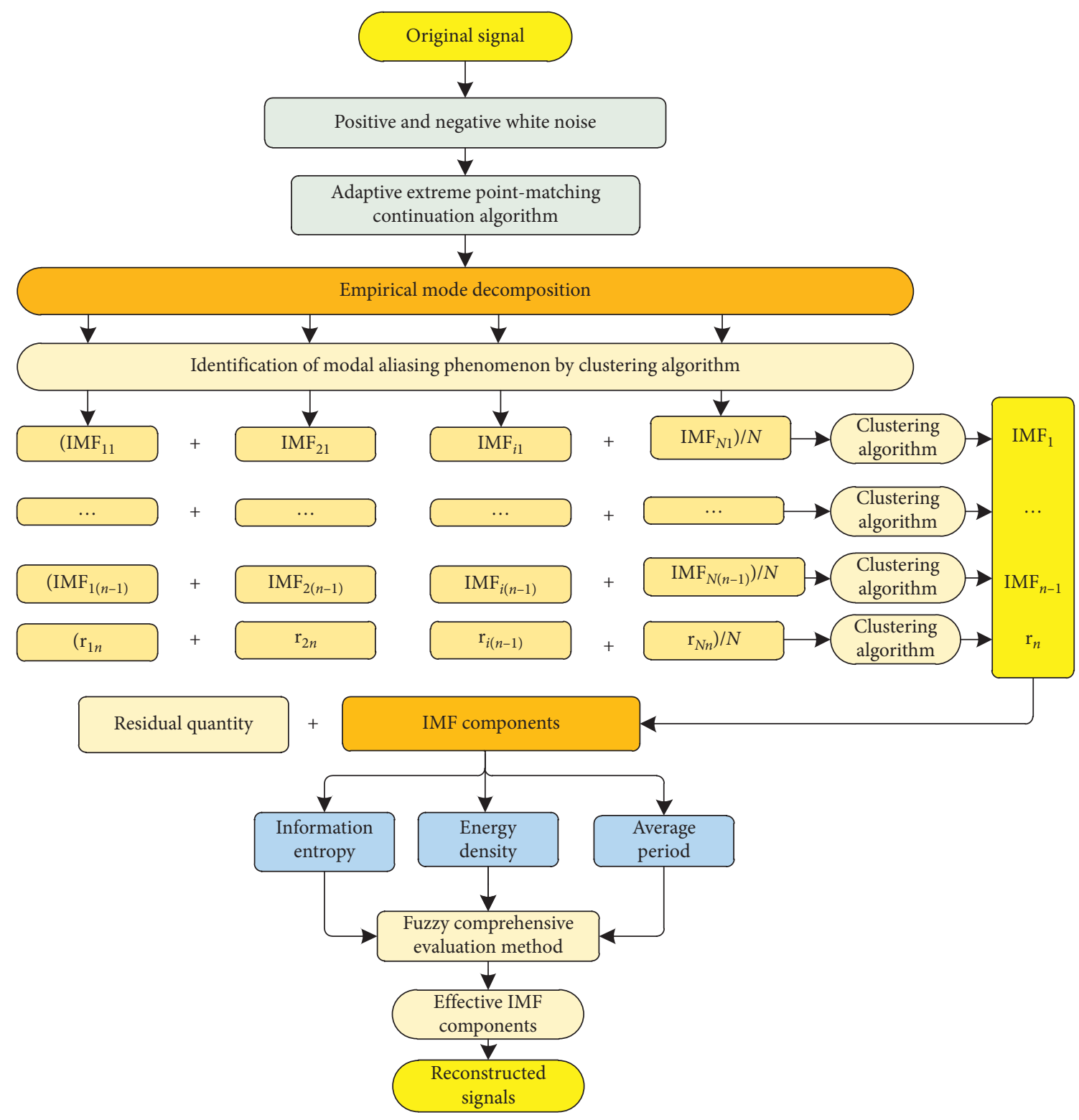

Figure 2: Flowchart of improved EEMD.

matrix $S$ with dimensions of $n \times l$. The following relationships exist among these matrices:

$$
\begin{aligned}
Q & =R \cdot \Lambda \cdot S^{T}, \\
\Lambda & =\operatorname{diag}\left(\lambda_{1}, \lambda_{2}, \ldots, \lambda_{l}\right) .
\end{aligned}
$$

An analysis of the principal diagonal elements $\lambda_{i}$ (singular values of the matrix $Q$ ) in matrix $\Lambda$ indicates that the number of the principal diagonal elements is closely related to the complexity of the frequency components contained in the signal. The more elements there are, the more complex the original signal components are. When the original signal is disturbed by noise, the principal diagonal elements are likely to be nonzero, which means that the frequency component of the original signal is relatively simple. According to this characteristic, the vibration signal information can be objectively reflected by the matrix $\Lambda$. The definition of singular entropy is as follows:

$$
\begin{aligned}
E_{k} & =\sum_{i=1}^{k} \Delta E_{i}, \quad k \leq l, \\
\Delta E_{i} & =-\left(\frac{\lambda_{i}}{\sum_{k=1}^{l} \lambda_{k}}\right) \ln \left(\frac{\lambda_{i}}{\sum_{k=1}^{l} \lambda_{k}}\right),
\end{aligned}
$$

where $k$ is the order of the singular entropy and $\Delta E_{i}$ is the increment of the singular entropy of the $i^{\text {th }}$ order.

As the order continuously increases, the rate of increase in the singular entropy gradually decreases and finally stabilizes. This characteristic does not change with noise level of the signal. Based on this relation, the change in singular entropy can be regarded as a criterion of the system order; that is, when the change in the singular entropy 
stabilizes, the corresponding order is the real order of the system. Therefore, in practice, a derivative of the change in singular entropy is often taken. When the derivative value approaches 0 , the change in singular entropy tends to stabilize, and the corresponding order can be regarded as the real order of the system.

3.2. Automatic Identification of Modal Parameters. There are two typical difficulties in modal parameter identification using the DATS-SSI algorithm: system order determination and physical mode selection. The traditional SSI method is based on the stability diagram and manual steps, which not only reduces the working efficiency but also leads to subjective differences in the identification results due to individual differences.

Based on this, this paper makes in-depth research on the relevant principles of a stabilized diagram. It is found that the modal parameters of a bridge structure will not change greatly in a short time. That is, the real modals will exist in both the stabilized diagram at this moment and the stabilized diagram at the next moment. Only the false modals will change, which is mainly because the response signals collected by the sensors at different times will be affected by different degrees of noise, but it will still contain the structural information of the bridge structure.

According to this property, the real modes of structures can be selected from the multiple stability diagrams. In this case, the structures that appear in the multiple diagrams are used. However, in practice, it is time consuming and labor intensive to identify the real modes manually and individually, and the selection of real modes is influenced by subjectivity due to the differences among individuals. Therefore, the hierarchical clustering method in statistics is introduced to perform the automatic selection of real modes and the automatic identification of modal parameters.

Detailed descriptions of the implementation steps of the modal parameter automatic identification algorithm based on improved EEMD and the hierarchical clustering analysis are given below.

3.2.1. Adaptive Decomposition and the Reconstruction of Structural Signals. The improved EEMD algorithm proposed in this paper is used for the adaptive decomposition and reconstruction of the structural response signals that are collected by the sensor. Assuming that the sensor has collected the response data for a bridge structure for a total of $N$ days, $N$ groups of reconstructed signals can be obtained in this period.

3.2.2. Determination of the System Order. The reconstructed signals are decomposed by singular values, and the real order of the bridge structure, which is represented by letter $n$, is determined using the algorithm in Section 2.1.

\subsubsection{Order Range Determination Based on the Stability} Diagram. The order range of the stability diagram is $[1,2 n]$, that is, the calculation starts from the first order of the system, and the maximum calculation order is $2 n$.
3.2.4. Modal Parameter Identification. The modal parameters of $N$ groups of reconstructed signals are identified by DATASSI, and $N$ groups of identification results are obtained. In addition, the results of each group include the corresponding frequency value, damping ratio, and mode coefficient.

3.2.5. Establishment of the Distance Matrix. It is assumed that $N$ groups of recognition results belong to a distinct category, that is, $N$ subsets can be established and are represented by $X_{1}, X_{2}, \ldots, X_{N}$. In addition, the results for each group include the corresponding frequency value, damping ratio, and mode coefficient. Starting from the first subset, the distance (similarity) between the $i^{\text {th }}$ subset and the $i+1^{\text {th }}$ subset is calculated in turn, and a distance matrix $D^{(i)}$ with dimensions of $n \times n$ is obtained, where $n$ is the real order of the system.

To establish the distance matrix, it is necessary to define the statistical magnitude that can reflect the distance between modes. Because the stability diagram is composed of natural frequencies, damping ratios, and mode shapes, these three parameters are used as clustering factors to define the distance $d_{i j}$ between mode $i$ and mode $j$. The calculation formula is as follows:

$$
d_{i j}=\frac{w_{f}}{d_{f}} \frac{\left|f_{i}-f_{j}\right|}{\max \left(f_{i}, f_{j}\right)}+\frac{w_{\xi}}{d_{\xi}} \frac{\left|\xi_{i}-\xi_{j}\right|}{\max \left(\xi_{i}, \xi_{j}\right)}+\frac{w_{\psi}}{d_{\psi}}(1-\operatorname{MAC}(i, j)),
$$

where $d_{f}, d_{\xi}$, and $d_{\psi}$ represent the frequency, damping ratio, and modal shape tolerances, respectively. According to the literature [19], the tolerance values are determined as $d_{f}=0.01, d_{\xi}=0.05$, and $d_{\psi}=0.02$, where $w_{f}, w_{\xi}$, and $w_{\psi}$ represent the frequency, damping ratio, and mode shape weights in the calculation of the modal distance. The sum of the three components is 1 , and in this paper, the values of these three variables are as follows: $w_{f}=0.4$, $w_{\xi}=0.3$, and $w_{\psi}=0.3$. The corresponding frequency weight value is larger than that of the other two variables because the focus of this paper is identifying the exact frequency value; therefore, the corresponding frequency value should be larger than the other two values, i.e., damping ratio and mode shape.

\subsubsection{Clustering of Similar Items}

(a) The distance matrix $D^{(k)}$ composed of the $k^{\text {th }}$ group and the $k+1^{\text {th }}$ group can be obtained via Section 3.2.5.

(b) The clustering of the same modes can be performed by determining the numerical values in the distance matrix. That is, when $d_{i j}^{(k)} \leq 1$, the $i^{\text {th }}$ mode of the $k^{\text {th }}$ group and the $j^{\text {th }}$ modal of the $k+1^{\text {th }}$ group have consistent frequency, damping ratio, and modal shape values. In other words, these two modes are considered to be of the same type. Therefore, $X_{k}(i)$ and $X_{k+1}(j)$ should be clustered together.

(c) All the modes of the same type in groups $k$ and $k+1$ should be clustered into one category, and the 
category should be used to obtain a new subset $X_{k+1}$ with the remainder of the modes.

(d) According to the same principle, the subsets $X_{k+1}$ and $X_{k+2}$ obtained in Section 3.2.3 are clustered, and a new subset $X_{k+2}$ is constructed. Then, this subset is clustered with subset $X_{k+3}$, and the process continues until all $N$ subsets are clustered and the final subset $X_{N}$ is obtained.

(e) The number of modal clustering elements of each order in the new subset $X_{N}$ is counted, and according to reference [19], if the number of clustering elements is greater than $0.6 n$ (where $n$ is the real order of the system), the mode is considered to be real, and a corresponding stability diagram is constructed.

The basic flowchart of the automatic identification of modal parameters for bridge structures based on the improved EEMD and hierarchical clustering analysis is shown in Figure 3.

\section{Verification of the Simulated Signal}

Both the improved EEMD algorithm and the EEMD algorithm are used to decompose the analog signals, and the results obtained by the two methods are compared. The analog signal is composed of sinusoidal signals of $1 \mathrm{~Hz}, 3 \mathrm{~Hz}$, and $7 \mathrm{~Hz}$ superimposed with stochastic noise (the noise level is approximately 10\%). The analog signal can be expressed as follows:

$$
s(t)=7 \sin (14 \pi t)+3 \sin (6 \pi t)+\sin (2 \pi t)+10 \text { rand } .
$$

The time of the analog signal is 10 seconds, and every 0.01 second represents one test point. There are a total of 1000 test points. The time domain diagram of the superimposed signal and noise is shown in Figure 4.

In the following section, the improved EEMD algorithm and the EEMD algorithm are used for the modal decomposition of the above analog signals. The specific analysis is as follows.

4.1. Comparison of the Endpoint Effect. The results of the IMF components corresponding to the two decomposition algorithms are shown in Figures 5 and 6, and the following conclusions can be obtained by comparing IMFs 3-5 in Figure 5 and IMFs 2-4 in Figure 6.

(1) Comparing the endpoint waveform shape of IMF3 (which is calculated by IEEMD algorithm) to IMF2 (which is calculated by EEMD algorithm), IFM4 (IEEMD) to IFM3 (EEMD), and IFM5 (IEEMD) to IFM4 (EEMD), it can be deduced that the improved EEMD algorithm can improve the endpoint effects of the decomposition results.

(2) Using the endpoint effect evaluation index in Section 2.2 for quantitative determination, it can be

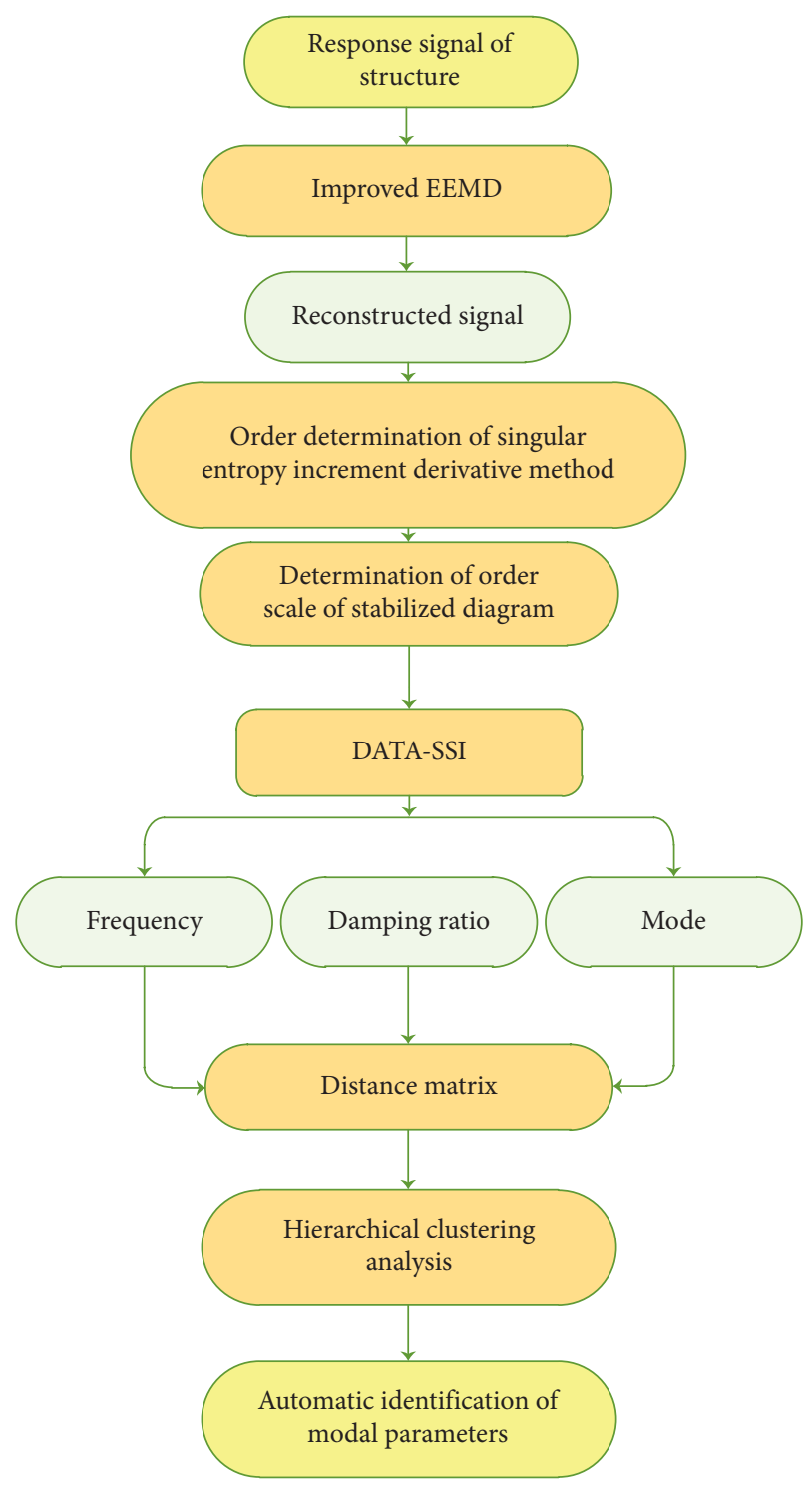

FIGURE 3: Flowchart of the automatic identification of modal parameters.

concluded that the evaluation index corresponding to the EEMD algorithm is 0.7358 and that of the adaptive extreme matching continuation method is 0.0084 .

Based on the above results, the adaptive extreme pointmatching algorithm proposed in this paper can improve the endpoint determination in the EEMD algorithm.

4.2. Mode Aliasing. To verify whether mode aliasing exists among the obtained IMF components, the clustering results corresponding to the IMF components of the two decomposition algorithms are presented and compared, as shown in Figures 7 and 8. The following conclusions can be drawn from the comparison of the results:

(1) Figure 7 shows that each IMF component is a separate category; that is, there is no similar information 


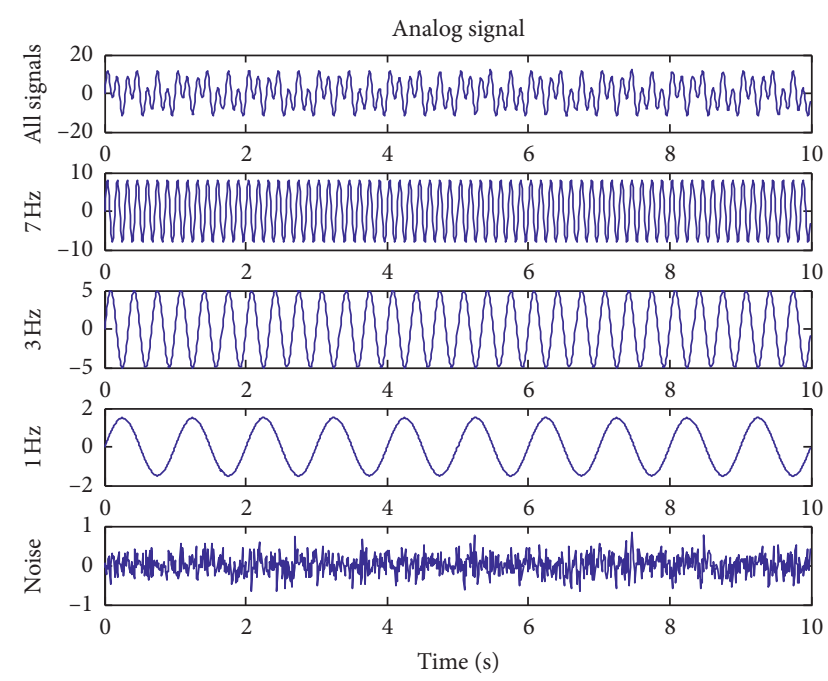

FIgURE 4: Signal and noise.
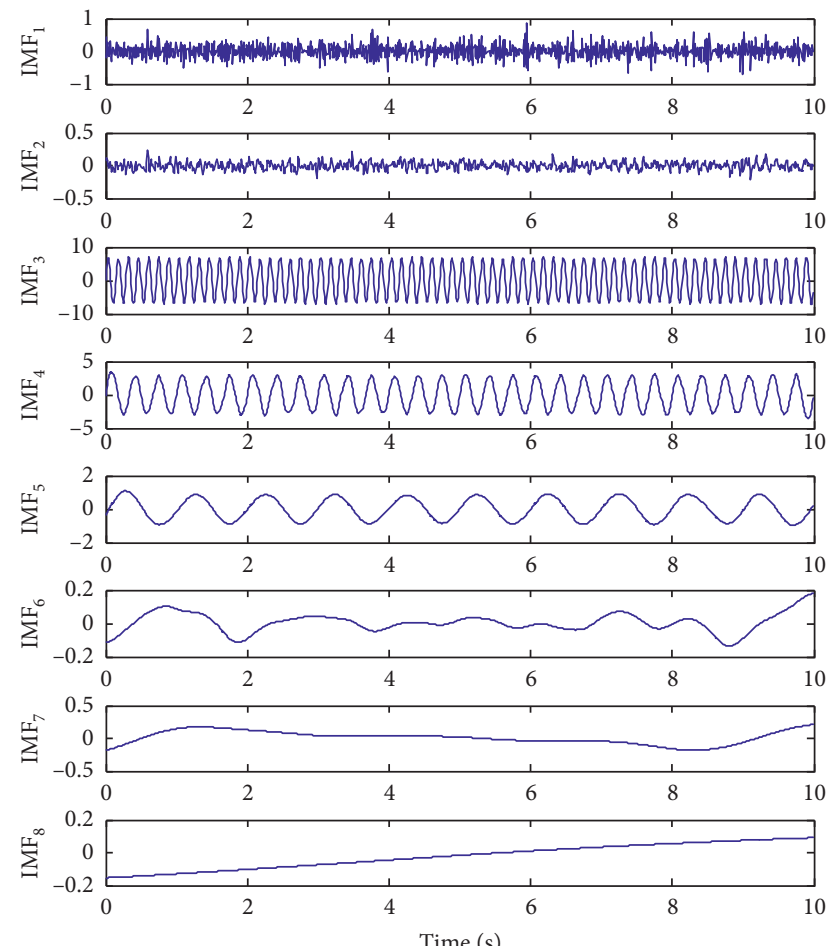

FIgURE 5: Results of decomposition (improved EEMD).

among the IMF components, which indicates that there is no mode aliasing among these IMF components.

(2) Figure 8 shows that $\mathrm{IMF}_{1}$ and $\mathrm{IMF}_{2}$ are classified in the same category and $\mathrm{IMF}_{5}$ and $\mathrm{IMF}_{6}$ are classified in the same category, which indicates that there is some similar information between these components, i.e., there is mode aliasing.

The above findings indicate that in the process of signal decomposition, the clustering analysis algorithm can be introduced to avoid mode aliasing.

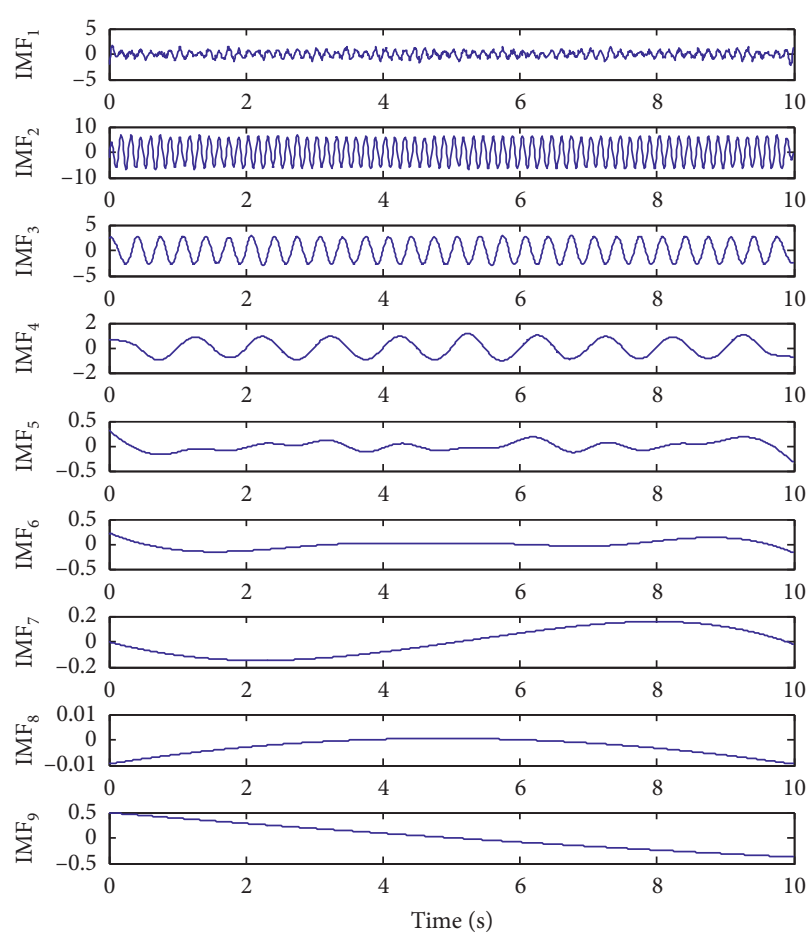

Figure 6: Results of decomposition (EEMD).

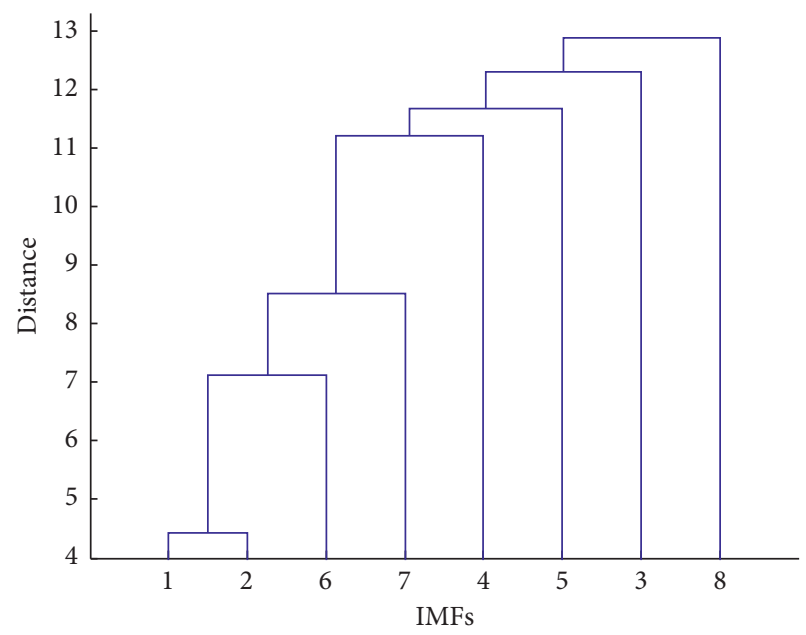

Figure 7: Results of clustering (improved EEMD).

4.3. Filtering of Effective IMF Components. A new comprehensive evaluation algorithm is proposed to solve the problem with the EEMD algorithm. Specifically, the EEMD algorithm cannot filter the effective IMF components. The information entropy, energy density, and average period of each IMF component are used to construct a screening index, which can be used to screen the effective IMF components. The specific analyses are shown in Tables 1 and 2. By comparing the data in the two tables, the following conclusions can be drawn:

(1) According to Table 1, the corresponding coefficients of effectiveness for $\mathrm{IMF}_{3}, \mathrm{IMF}_{4}$, and $\mathrm{IMF}_{5}$ are greater 


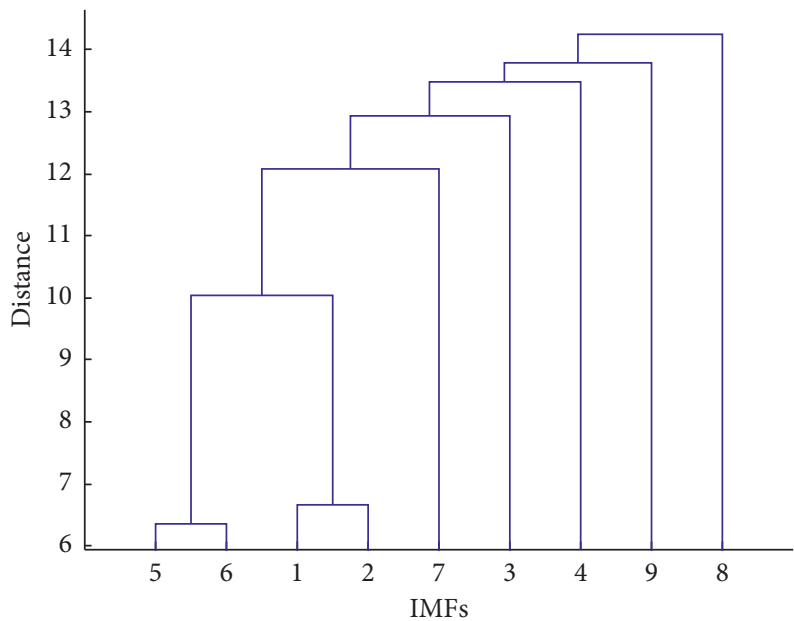

Figure 8: Results of clustering (EEMD).

TABLE 1: Table of effectiveness degree coefficients (improved EEMD).

\begin{tabular}{|c|c|c|c|c|c|c|c|c|}
\hline Indicators & $\mathrm{IMF}_{1}$ & $\mathrm{IMF}_{2}$ & $\mathrm{IMF}_{3}$ & $\mathrm{IMF}_{4}$ & $\mathrm{IMF}_{5}$ & $\mathrm{IMF}_{6}$ & $\mathrm{IMF}_{7}$ & $\mathrm{IMF}_{8}$ \\
\hline Information entropy & 2.66 & 2.32 & 0.31 & 0.32 & 0.34 & 0.99 & 0.97 & 0.57 \\
\hline Effectiveness coefficient & 0.12 & 0.45 & 1.00 & 1.00 & 0.86 & 0.01 & 0.22 & 0.02 \\
\hline Effectiveness degree coefficient & 0.00 & 0.31 & 1.00 & 1.00 & 0.93 & 0.36 & 0.47 & 0.25 \\
\hline
\end{tabular}

TABle 2: Table of effectiveness degree coefficients (EEMD).

\begin{tabular}{lccccccccc}
\hline Indicators & $\mathrm{IMF}_{1}$ & $\mathrm{IMF}_{2}$ & $\mathrm{IMF}_{3}$ & $\mathrm{IMF}_{4}$ & $\mathrm{IMF}_{5}$ & $\mathrm{IMF}_{6}$ & $\mathrm{IMF}_{7}$ & $\mathrm{IMF}_{8}$ & $\mathrm{IMF}_{9}$ \\
\hline Information entropy & 2.73 & 0.33 & 0.43 & 0.70 & 1.79 & 1.69 & 1.35 & 1.49 & 1.02 \\
Effectiveness coefficient & 0.23 & 1.00 & 0.78 & 0.86 & 0.21 & 0.12 & 0.08 & 0.03 & 0.01 \\
Effectiveness degree coefficient & 0.11 & 1.00 & 0.87 & 0.85 & 0.30 & 0.27 & 0.32 & 0.27 & 0.36 \\
\hline
\end{tabular}

than 0.8; thus, all these components are effective IMF components that can be used to reconstruct the signals.

(2) According to Table 2, the corresponding coefficients of effectiveness for $\mathrm{IMF}_{2}, \mathrm{IMF}_{3}$, and $\mathrm{IMF}_{4}$ are greater than 0.8 ; therefore, all these components are effective IMF components that can be used to reconstruct the signals.

(3) An analysis of the filtered IMF components based on the decomposition diagrams shown in Figures 5 and 6 indicates that the comprehensive evaluation algorithm proposed in this paper can screen out the corresponding signals of $7 \mathrm{~Hz}, 3 \mathrm{~Hz}$, and $1 \mathrm{~Hz}$ from the superimposed signals. Thus, this algorithm can automatically filter the effective IMF components.

However, to further verify that the improved EEMD algorithm can better handle endpoint effects, the instantaneous frequencies corresponding to the effective IMF components in the results of these two decomposition algorithms are given, as shown in Figures 9 and 10. A comparison of the two figures suggests that both decomposition algorithms can highlight the frequency components

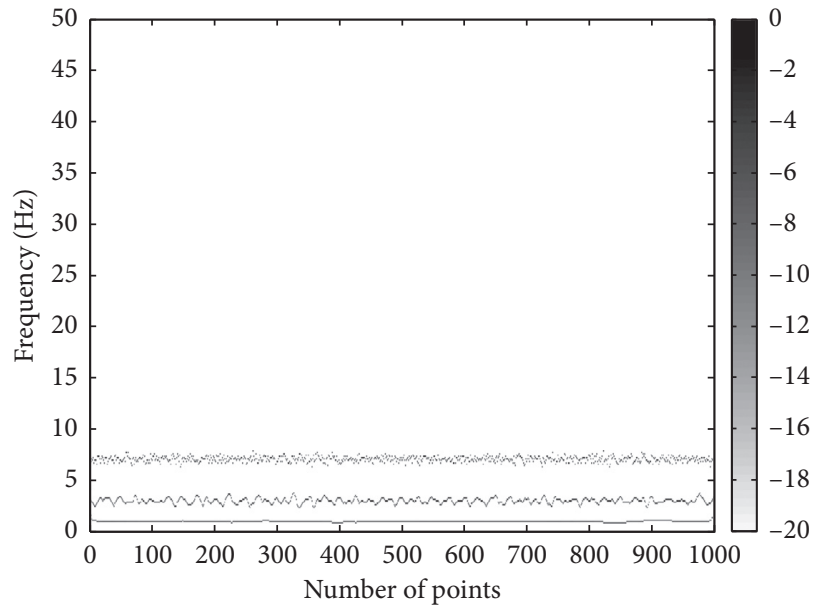

Figure 9: Hilbert-Huang plot (improved EEMD).

of analog signals. Based on the change in the instantaneous frequency at the endpoint, the improved EEMD algorithm proposed in this paper can better handle endpoint effects compared to the traditional EEMD method. 


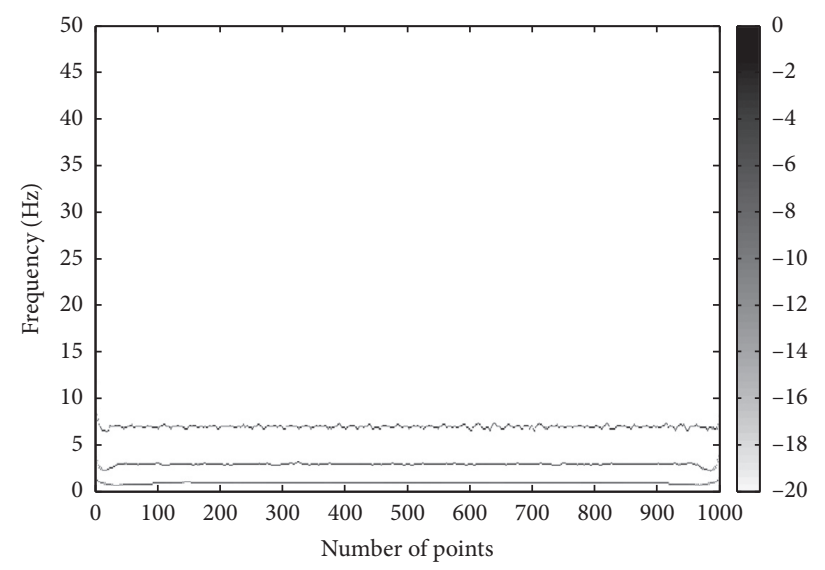

FIgURE 10: Hilbert-Huang plot (EEMD).

\section{Automatic Identification of Modal Parameters for Actual Bridge Structures}

In this paper, the modal parameters for Sutong Bridge are automatically identified. The specific steps are as follows:

(1) First, the structural response signals collected by sensors are analyzed with an exploratory data analysis method, and the structural signals satisfying a normal distribution are screened and removed.

(2) Second, the improved EEMD algorithm is used to decompose and reconstruct the acceleration response signal.

(3) Third, the reconstructed signal is decomposed based on a singular value, and the system order is determined according to the algorithm proposed in Section 2.1.

(4) Fourth, the DATA-SSI algorithm is used to identify the modal parameters of the reconstructed response signal, and various group identification results are obtained.

(5) Fifth, the automatic identification of modal parameters is performed using the algorithm introduced in Section 2.2.

(6) Finally, the automatic identification results are compared with the real results to verify the feasibility of the proposed algorithm.

5.1. Project Profile. The Sutong Bridge shown in Figure 11 is a large cable-stayed bridge. The main span is a $108 \mathrm{~m}$ cablestayed bridge with twin towers and a cable plane. The bridge is located on the Yangtze River. The total length of the bridge structure is $2088 \mathrm{~m}$, and the specific span arrangement is $(2 \times 100+300+1088+300+2 \times 100) \mathrm{m}$.

Because the bridge is a large cable-stayed bridge, a complete health monitoring system was designed during construction to constantly monitor the operation status of the bridge and evaluate its performance. There are many types of sensors on the bridge, including temperature sensors, cable force sensors, and acceleration sensors with a sampling frequency of $20 \mathrm{~Hz}$. In this paper, the vertical response signals measured by 14 acceleration sensors on the main bridge are selected for modal parameter identification. The layout of the acceleration sensors is shown in Figure 12.

5.2. Filtering of the Structural Response Signal. The health monitoring system of the bridge structure can continuously collect data collection, and large datasets are available. If the response signals for an entire year need to be analyzed, then the workload will be very large. In view of this limitation, this paper uses a quarterly approach, and only one representative month in each quarter is selected for investigation and parameter change analysis. The selected months are March (spring), July (summer), October (autumn), and December (winter). The histogram method of exploratory data analysis is used to analyze the acceleration response signals in the above four months. Figure 13 shows the corresponding histograms of the response signals collected by 14 sensors in July. According to the figure, the structural response signals collected by the second sensor do not satisfy a normal distribution, and only the response signals collected by the other 13 sensors can be used for modal decomposition and parameter identification.

5.3. Order-Based Implementation of the Incremental Singular Entropy Method. The improved EEMD algorithm is used to decompose and reconstruct the acceleration response signals collected by the sensors. Then, the singular values of the reconstructed signals are decomposed, and the corresponding singular entropy is calculated to obtain the singular entropy variations as the order increases, as shown in Figure 14. To determine whether the singular entropy stabilizes, the first-order derivative of the change in the singular entropy is obtained, as shown in Figure 15.

Figures 14 and 15 show that the change in the singular entropy stabilizes, and the first-order derivative approaches zero when the system order is 150; that is, the real order of the bridge structure is considered to be 150 . 


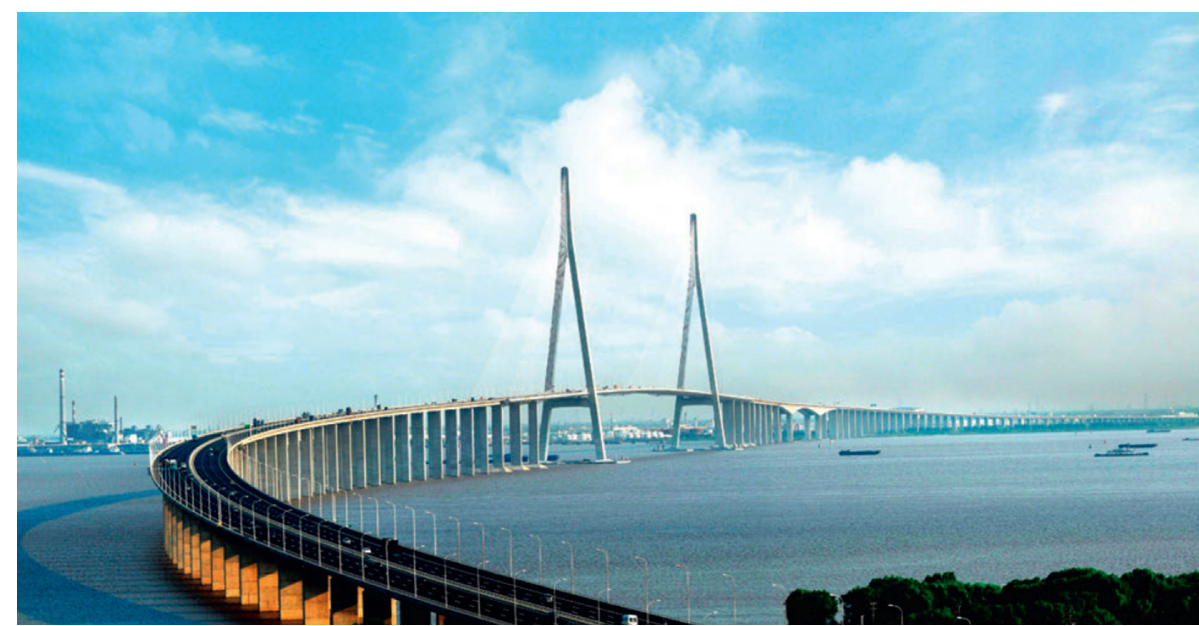

Figure 11: Photography of Sutong Bridge.

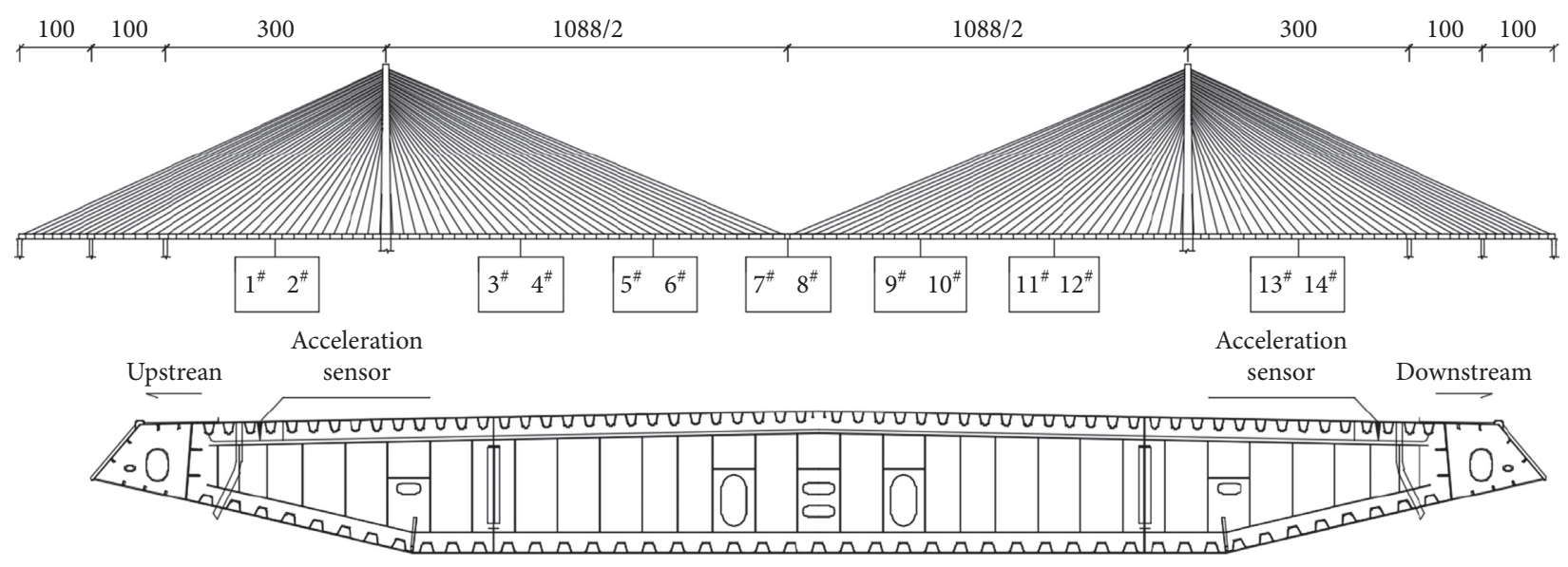

FiguRE 12: Acceleration sensor arrangement.

5.4. Automatic Identification of Modal Parameters. To verify that the improved EEMD algorithm proposed in this paper can decompose and reconstruct the signals of a real bridge better than the EEMD algorithm, the corresponding acceleration response signals on July 1, 2018, were selected for analysis, and the above two decomposition algorithms were used to decompose and reconstruct the response signals separately. Then, the obtained reconstructed signals were used as inputs to the DATA-SSI algorithm, leading to two stability diagrams, as shown in Figures 16 and 17. In this figure, the red color represents the stable frequency, blue color represents the stable damping ratio, and green color represents the stable mode shape.

A comparison of the two figures suggests that the signals that are processed by the improved EEMD algorithm result in a stability diagram with few false modes. The stability axis is much clearer, especially between $3 \mathrm{~Hz}$ and $5 \mathrm{~Hz}$. Therefore, compared with the EEMD algorithm, the improved EEMD algorithm can extract more accurate structural information.

However, it is difficult to select the real modes of the bridge structure according to Figures 16 and 17 because there are many false modes in the figures, and there is no criterion for distinguishing the true modes from the false modes. The corresponding final stability diagram for each month can be obtained as follows:

(1) In units of days, the improved EEMD algorithm was used to decompose and reconstruct the acceleration response signals each day, and the reconstructed signals were retained. There were 31 groups in total.

(2) The DATA-SSI algorithm was used to identify the modal parameters of each group of reconstructed signals, and the results were retained, including the frequency values, damping ratios, and modal shapes.

(3) According to the theory introduced in Section 2.2, 31 groups of recognition results were analyzed based on hierarchical clustering analysis, which can automatically select the real modes and be used to construct the final stability diagram.

According to the above steps, the automatic identification of modal parameters can be performed. The corresponding stability diagram of the bridge structure in July is given, as shown in Figure 18. The proposed automatic identification algorithm for modal parameters, which is 

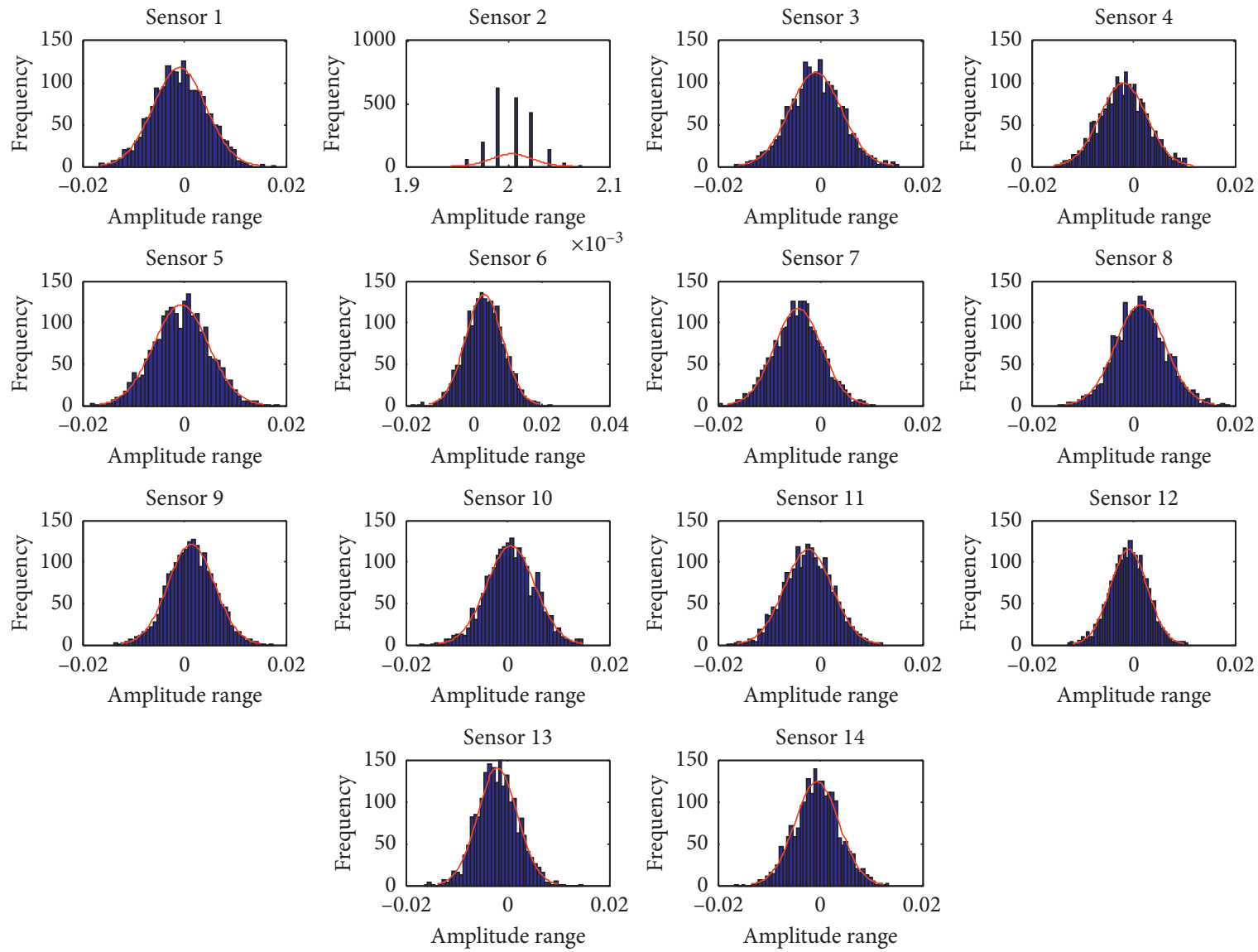

Figure 13: Data histograms.

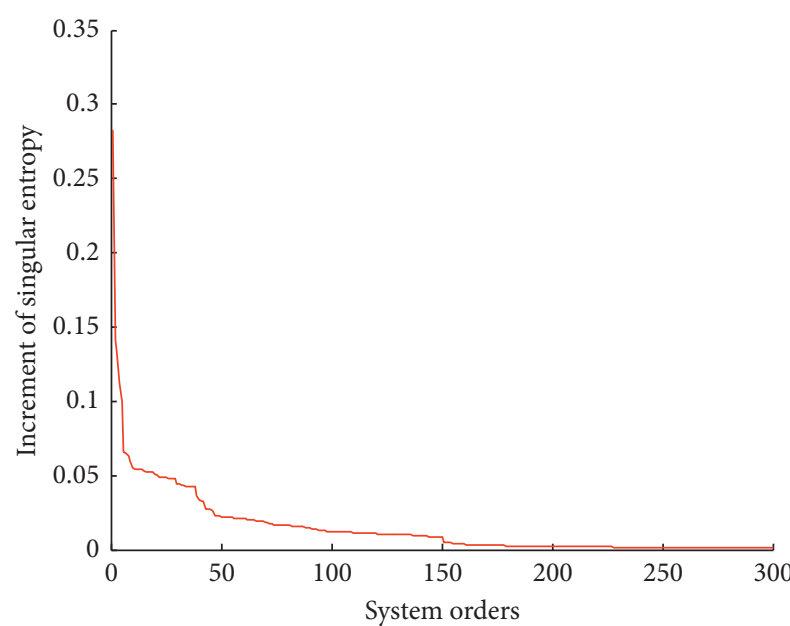

FIgURE 14: The trend of the change in singular entropy.

based on the improved EEMD and hierarchical clustering analysis, can eliminate the false modes and preserve the real modes. To further verify that the identified parameter values are similar to the real parameter values of the bridge structure, the top five frequency values obtained from the identification are compared with the calculated values based on the theoretical vertical frequency, which is given in reference [23].

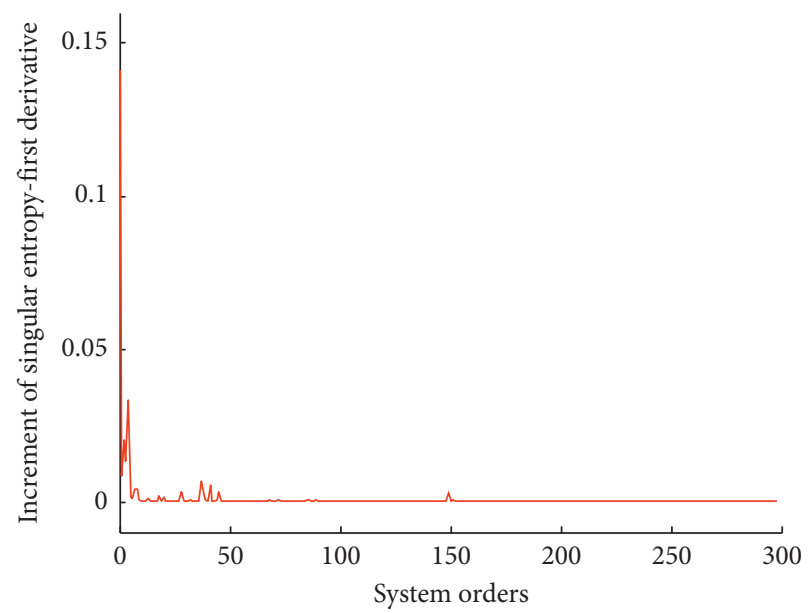

Figure 15: The slope trend of the change in singular entropy.

Table 3 shows the comparison between the results obtained by improved EEMD and the real values in reference [23], and Table 4 shows the comparison between the results obtained by EEMD and the real values in reference [23]. Analyzing the data in two tables, we know that the results from improved EEMD is better than results from EEMD, and the improved EEMD can be used for the automatic identification of modal parameters for the actual bridge structure, and the frequency values based 


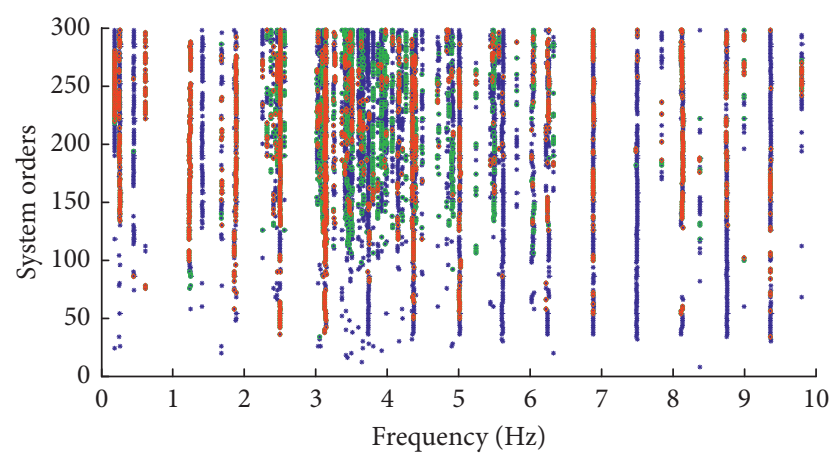

FIgURE 16: Stability diagram of the improved EEMD method.

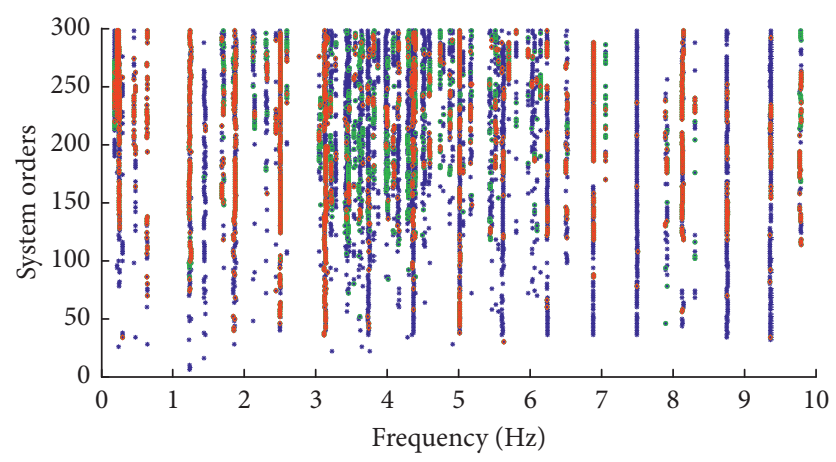

FIgURE 17: Stability diagram of EEMD.

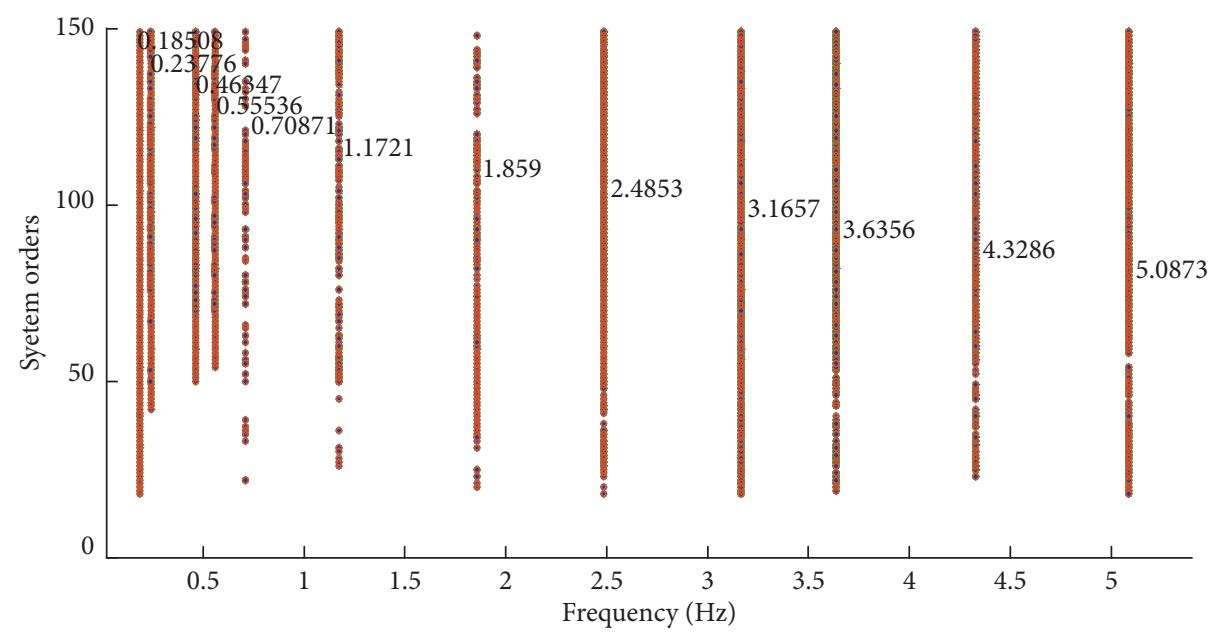

FIGURE 18: Stability diagram of hierarchical clustering (July).

TABLE 3: Comparison between improved EEMD results and real values $(\mathrm{Hz})$.

\begin{tabular}{lcccccccc}
\hline Model order & True value [23] & March & July & October & December & Average & Tolerance & Percentage difference (\%) \\
\hline First & 0.1856 & 0.1845 & 0.1851 & 0.1845 & 0.1849 & 0.1847 & 0.0009 & 0.46 \\
Second & 0.2191 & 0.2217 & 0.2378 & 0.2200 & 0.2378 & 0.2293 & 0.0102 & 3.22 \\
Third & 0.4531 & 0.4624 & 0.4635 & 0.4634 & 0.4644 & 0.4634 & 0.0103 & 2.28 \\
Fourth & 0.5746 & 0.5542 & 0.5554 & 0.5546 & 0.5389 & 0.5508 & 0.0238 & 4.15 \\
Fifth & 0.6928 & 0.6911 & 0.7087 & 0.7062 & 0.6858 & 0.6979 & 0.0051 & 0.74 \\
\hline
\end{tabular}


TABLE 4: Comparison between EEMD results and real values $(\mathrm{Hz})$.

\begin{tabular}{lcccccccc}
\hline Model order & True value [23] & March & July & October & December & Average & Tolerance & Percentage difference (\%) \\
\hline First & 0.1856 & 0.1914 & 0.1791 & 0.1736 & 0.1815 & 0.1711 & 0.0063 & 3.37 \\
Second & 0.2191 & 0.2017 & 0.2164 & 0.2684 & 0.2711 & 0.2064 & -0.0137 & -6.25 \\
Third & 0.4531 & 0.4208 & 0.4311 & 0.4217 & 0.4319 & 0.3754 & 0.0369 & 8.15 \\
Fourth & 0.5746 & 0.5099 & 0.5054 & 0.5158 & 0.4904 & 0.5398 & 0.0624 & 10.85 \\
Fifth & 0.6928 & 0.7602 & 0.7370 & 0.7415 & 0.7064 & 0.7677 & -0.0498 & -7.18 \\
\hline
\end{tabular}

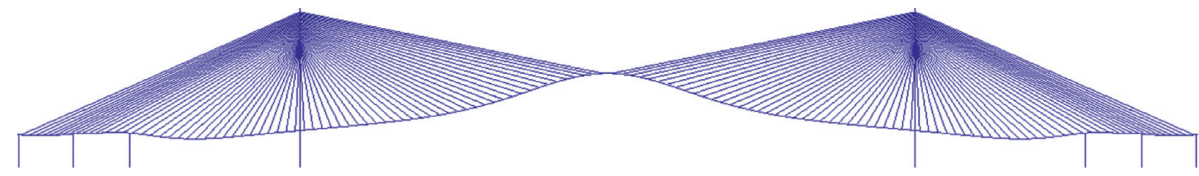

(a)

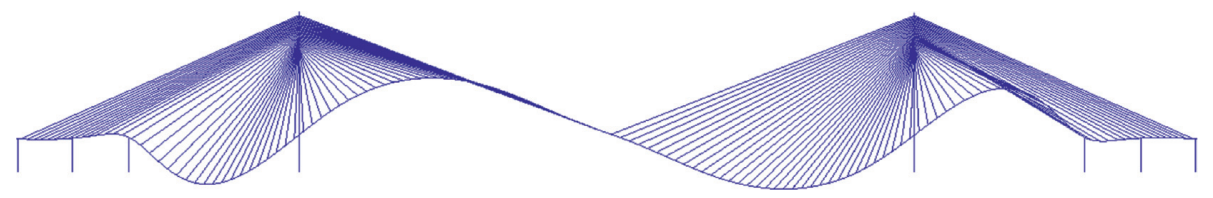

(b)

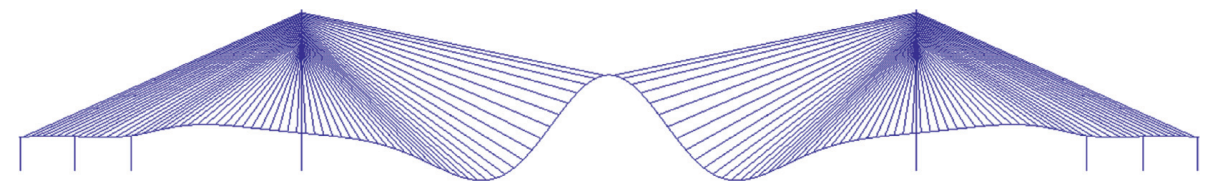

(c)

FIgURE 19: Modal shape diagrams of the top three orders (MIDAS model). (a) The first-order mode shape. (b) The second-order mode shape. (c) The third-order mode shape.

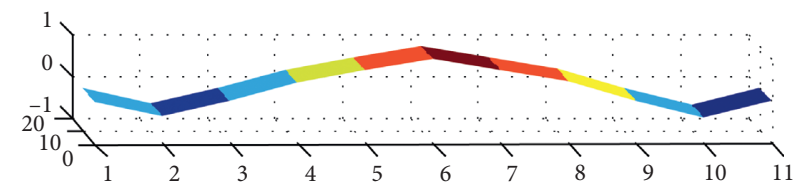

(a)

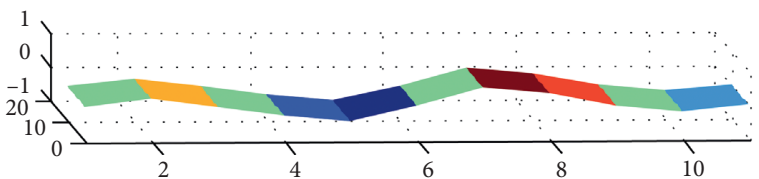

(b)

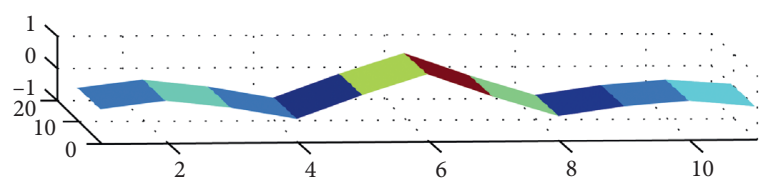

(c)

Figure 20: Modal shape diagrams of the top three orders (algorithm of this paper). (a) The first-order mode shape. (b) The second-order mode shape. (c) The third-order mode shape.

on identification are very similar to the values calculated based on theory, with an error of less than 5\%.

MIDAS software was used for modelling of bridge and the top three modes were obtained as shown in Figure 19, and Figure 20 is a modal shape diagram of the cable-stayed bridge for the top three orders identified by the proposed algorithm. Comparing the two results, we can know that this diagram is very similar to the actual modal diagram, with a similarity of approximately $95 \%$, which further verifies the feasibility of the proposed algorithm.
5.5. Quarterly Frequency Changes in the Longitudinal Direction of the Bridge. To study the specific frequency value trends of the top five orders in the longitudinal direction of the bridge structure in each quarter, the corresponding frequency values in March, July, October, and December were identified. In total, 124 days in these four months were studied, and the frequency value trends of each order over time are shown in Figure 21. The frequency values of the first and second orders are relatively stable in 2018 for the bridge structure; however, the values of the other three orders 


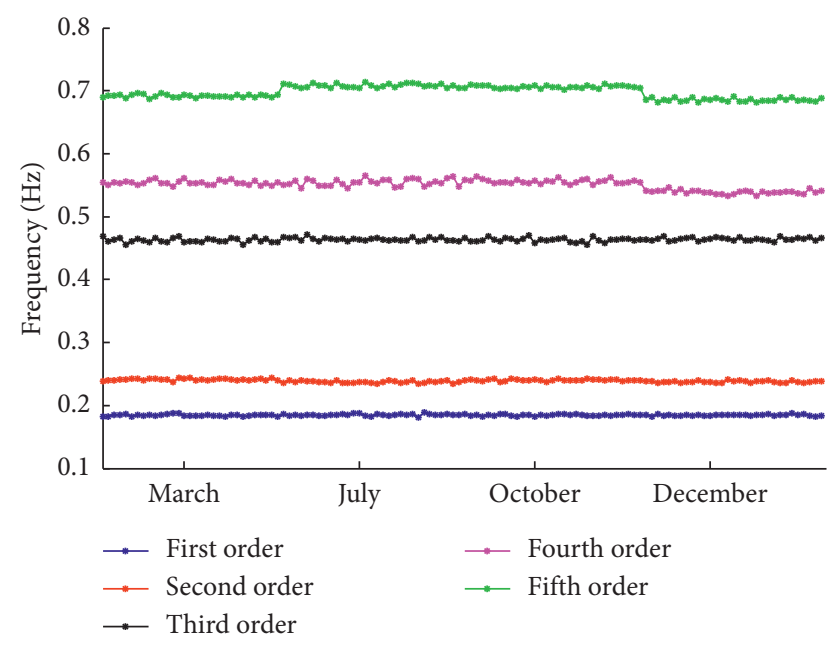

Figure 21: Frequency value trends for the top five orders.

fluctuate with time, but within a small range. Because the modal parameters of the bridge structure can reflect the inherent dynamic characteristics of the bridge structure and these characteristics did not obviously change in 2018, the bridge structure was in a healthy and stable state.

\section{Conclusion}

The following conclusions were obtained by applying the proposed algorithm to the analog signals and measured data for an actual bridge:

(1) The proposed adaptive extreme point-matching continuation algorithm can improve the endpoint effect problems in the EEMD algorithm.

(2) When clustering analysis is applied in the process of modal decomposition, the mode aliasing among the obtained IMFs can be avoided.

(3) The effectiveness degree coefficient, a new index constructed based on the information entropy, energy density, and average period, can be used to automatically select the effective IMF components.

(4) By taking the derivative of the singular entropy change, the real order of a system can be automatically determined when identifying the modal parameters of an actual bridge structure.

(5) By introducing the hierarchical clustering analysis algorithm, the real modes in the stability diagram can be automatically selected, and the modal parameters can be automatically identified.

(6) The processing results based on test data for actual bridges indicate that the proposed automatic identification algorithm of modal parameters for bridge structures based on improved EEMD and hierarchical clustering analysis can effectively perform the adaptive decomposition and reconstruction of structural response signals and the automatic determination of the system order and modal parameters.

\section{Data Availability}

The data used to support the findings of this paper are freely available. It can be made available on request, if and when required.

\section{Conflicts of Interest}

The authors declare that they have no conflicts of interest.

\section{Acknowledgments}

This work was funded by the National Basic Research Program of China (no.2013CB036302).

\section{References}

[1] F. Magalhães, Á. Cunha, and E. Caetano, "Online automatic identification of the modal parameters of a long span arch bridge," Mechanical Systems and Signal Processing, vol. 23, no. 2, pp. 316-329, 2009.

[2] R. J. Allemang, D. L. Brown, and A. W. Phillips, "Survey of modal techniques applicable to autonomous/semi-autonomous parameter identification," in Proceedings of the International Conference on Noise and Vibration Engineering (ISMA'10), p. 42, Katholieke Universiteit Leuven, Leuven, Belgium, September 2010.

[3] R. J. Allemang, A. W. Phillips, and D. L. Brown, "Autonomous modal parameter estimation: statisical considerations," in Proceedings of the 29th IMAC Conference on Structural Dynamics (IMAC'11), pp. 385-401, Leuven, Belgium, February 2011.

[4] E. Ntotsios, C. Papadimitriou, P. Panetsos, G. Karaiskos, K. Perros, and P. C. Perdikaris, "Bridge health monitoring system based on vibration measurements," Bulletin of Earthquake Engineering, vol. 7, no. 2, pp. 469-483, 2008.

[5] N. E. Huang, M.-L. C. Wu, S. R. Long et al., "A confidence limit for the empirical mode decomposition and Hilbert spectral analysis," Proceedings of the Royal Society of London. Series A: Mathematical, Physical and Engineering Sciences, vol. 459, no. 2037, pp. 2317-2345, 2003.

[6] M. E. Torres, M. A. Colominas, G. Schlotthauer, and P. Flandrin, "A complete ensemble empirical mode decomposition with adaptive noise," in Proceedings of the 36th IEEE International Conference on Acoustics, Speech, and Signal Processing, pp. 4144-4147, Prague, Czech Republic, May 2011.

[7] B. Moaveni and E. Asgarieh, "Deterministic-stochastic subspace identification method for identification of nonlinear structures as time-varying linear systems," Mechanical Systems and Signal Processing, vol. 31, no. 8, pp. 40-55, 2012.

[8] L. Mingliang, W. Keqi, S. Laijun, and Z. Jianju, "Applying empirical mode decomposition (EMD) and entropy to diagnose circuit breaker faults," Optik, vol. 126, no. 20, pp. 2338-2342, 2015.

[9] L. Han, C. Li, and H. Liu, "Feature extraction method of rolling bearing fault signal based on EEMD and cloud model characteristic entropy," Entropy, vol. 17, no. 12, pp. 6683-6697, 2015.

[10] T. Wang, X. Wu, T. Liu, and Z. M. Xiao, "Gearbox fault detection and diagnosis based on EEMD De-noising and power spectrum," in Proceedings of the IEEE International Conference on Information and Automation (ICIA'15), pp. 1528-1531, IEEE, Lijiang, China, August 2015. 
[11] J. Doucette, Advances on design and analysis of mesh-restorable networks, Ph.D. thesis, University of Alberta, Edmonton, Canada, 2004.

[12] M. I. S. Bezerra, Y. Iano, and M. H. Tarumoto, "Evaluating some Yule-Walker methods with the maximum-likelihood estimator for the spectral ARMA model," Tendências em Matemática Aplicada e Computacional, vol. 9, no. 2, pp. 175-184, 2008.

[13] H. P. Graf, E. Cosatto, L. Bottou, I. Dourdanovic, and V. Vapnik, "Parallel support vector machines: the cascade svm," in Advances in Neural Information Processing Systems, L. K. Saul, Y. Weiss, and L. Bottou, Eds., vol. 17, pp. 521-528, MIT Press, Cambridge, MA, USA, 2005.

[14] M. A. Sanchez, O. Castillo, and J. R. Castro, "Generalized type-2 fuzzy systems for controlling a mobile robot and a performance comparison with interval type-2 and type-1 fuzzy systems," Expert Systems with Applications, vol. 42, no. 14, pp. 5904-5914, 2015.

[15] S. V. Guttula, A. Allam, and R. S. Gumpeny, "Analyzing microarray data of Alzheimer's using cluster analysis to identify the biomarker genes," International Journal of Alzheimer's Disease, vol. 2012, Article ID 649456, 5 pages, 2012.

[16] Y. Y. Chen and Y. M. Chen, "Attribute reduction algorithm based on information entropy and ant colony optimization," Journal of Chinese Computer System, vol. 36, no. 3, pp. 586590, 2015.

[17] X. L. Wang, L. Y. Lu, and W. P. Tai, "Research of a new algorithm of words similarity based on information entropy," Computer Technology and Development, vol. 25, no. 9, pp. 119-122, 2015.

[18] J.-F. Chen, H.-N. Hsieh, and Q. H. Do, "Evaluating teaching performance based on fuzzy AHP and comprehensive evaluation approach," Applied Soft Computing, vol. 28, pp. 100$108,2015$.

[19] Z. Song, H. Zhu, G. Jia, and C. He, "Comprehensive evaluation on self-ignition risks of coal stockpiles using fuzzy AHP approaches," Journal of Loss Prevention in the Process Industries, vol. 32, no. 1, pp. 78-94, 2014.

[20] Y.-N. Han, W. Zhou, and X.-Q. Zhang, "Fuzzy comprehensive evaluation of the adaptability of an expressway system," Journal of Highway and Transportation Research and Development (English Edition), vol. 8, no. 4, pp. 97-103, 2014.

[21] G. Zhang, B. Tang, and G. Tang, "An improved stochastic subspace identification for operational modal analysis," Measurement, vol. 45, no. 5, pp. 1246-1256, 2012.

[22] A. C. Altunişik, A. Bayraktar, and B. Sevim, "Operational modal analysis of a scaled bridge model using EFDD and SSI methods," Indian Journal of Engineering and Materials Sciences, vol. 19, no. 5, pp. 320-330, 2012.

[23] I. Khan, D. Shan, and Q. Li, "Modal parameter identification of cable stayed bridge based on exploratory data analysis," Archives of Civil Engineering, vol. 61, no. 2, pp. 3-22, 2015. 